\title{
Creación del Programa de Educación Continua de la Universidad de Costa Rica, Sede de Occidente: Retos y Logros
}

\author{
Creation of the Continuing Education Program of University of \\ Costa Rica, Western Campus: Challenges and Achievements
}

\author{
María Antonieta Vargas Solís* \\ Carolina Álvarez Fuentes**
}

\begin{abstract}
RESUMEN
Este artículo es una sistematización de experiencias sobre el Programa de Educación Continua de la Sede de Occidente (PECSO) de la Universidad de Costa Rica. El objetivo es dar a conocer los retos y logros del primer año de trabajo. Para la metodología se utilizaron cinco tiempos: Punto de Partida, Preguntas Iniciales, Recuperación del Proceso Vivido, Reflexiones de Fondo y Puntos de Llegada. En las reflexiones de fondo se explicaron por qué pasaron los cambios en cuanto a la ubicación geográfica, población meta del proyecto, los objetivos del programa y asuntos de gestión administrativa. El mayor logro del PECSO fue ofertar más cursos de los establecidos en los objetivos y su mayor reto fue lidiar con la gestión administrativa que conlleva abrir un programa de este tipo.
\end{abstract}

Palabras clave: educación continua, acción social, proyecto de extensión, actualización profesional

\begin{abstract}
This article is a systematization of experiences on the Continuing Education Program of the Western Headquarters of the University of Costa. The objective is to present the challenges and achievements of the first year of work. Five steps were used for the methodology: Departure Point, Initial Questions, Rebuilding the lived Process, Reflection, and Arrival Points. In the reflection step, it is explained why the changes occurred in terms of geographic location, project target population, program objectives, and administrative management issues. The greatest achievement of the program was to offer more courses than those established in the objectives, and its greatest challenge was to deal with the administrative management involved in opening such a program.
\end{abstract}

Key Words: continuing education, social action, community outreach project, professional development

\footnotetext{
* Universidad de Costa Rica. Docente Departamento de Educación, Sede de Occidente. Costa Rica. Correo electrónico: antonieta_vargas@ hotmail.com, ORCID: 0000-0003-0385-0660

** Universidad de Costa Rica. Docente Departamento de Educación, Sede de Occidente. Costa Rica. Correo electrónico: carolinaucr@ hotmail.com, ORCID: 0000-0002-0120-828X
}

Recepción: 20/12/2019 Aceptación: 19/05/2020 Doi: 10.15517/WL.V15I1.42010 
O人лмв Lu Wimblu, Rev. Estud. de Psicología UCR, 15(1) 2020 (Enero-Junio): 73-96 /ISSN: 1659-2107

\section{Introducción}

La presente sistematización de experiencias tiene como objetivo dar a conocer los retos y logros del primer año del Programa de Educación Continua de la Sede de Occidente (PECSO) de la Universidad de Costa (UCR). Resulta necesario realizar este trabajo para comprender el contexto que determinó la creación del PECSO y para establecer la tendencia evolutiva que se debe seguir.

La palabra sistematización, utilizada en diversas disciplinas, se refiere principalmente a clasificar, ordenar o catalogar datos e informaciones, a "ponerlos en sistema"...

Sin embargo, en el campo de la educación popular y de trabajo en procesos sociales, lo utilizamos en un sentido más amplio, referido no sólo a datos o informaciones que se recogen y ordenan, sino a obtener aprendizajes críticos de nuestras experiencias. Por eso, no decimos sólo "sistematización", sino "sistematización de experiencias". Jara (2011)

En este caso, la sistematización ordena la información y datos recolectados durante el proceso de apertura del Programa de Educación Continua en su primer año. Se expone un recuento histórico de cómo surge el programa con el fin de obtener aprendizajes de la experiencia vivida y así ayudar a otros funcionarios universitarios en la apertura de programas de índole similar, bajo el marco de la acción social.

Para sistematizar esta experiencia de acción social se tomaron en cuenta los 5 tiempos propuestos por Jara (2011), a saber: 1. el punto de partida, 2. las preguntas iniciales, 3. la recuperación del proceso vivido, 4. la reflexión de fondo y 5. los puntos de llegada. El primer punto busca registrar la experiencia vivida desde el punto de vista de la encargada y la colaboradora del programa. En las preguntas iniciales se pretende definir y delimitar el objeto de estudio, así como el procedimiento de sistematización. En el punto 3, se ordena y clasifica la información. En el punto 4, se hace una interpretación del proceso vivido y en el punto 5 se formulan las conclusiones. Así las cosas, se trata no solamente de describir el proceso sino de aprender de este.

Lo anterior por cuanto, no es extraño que los proyectos de acción o extensión social, como se les conoce en diferentes universidades, son creados o iniciados por docentes que tienen poco o nada de experiencia fuera de la docencia. Por lo que sistematizar esta 
O人ıмв Lu Wimblu, Rev. Estud. de Psicología UCR, 15(1) 2020 (Enero-Junio): 73-96 /ISSN: 1659-2107

experiencia era la mejor manera de compartir una práctica que puede llegar a mejorar los procesos en otras instituciones u organizaciones sobre todo de corte terciario.

Es importante conocer estos datos con el fin de motivar a otras entidades a promover programas de educación continua, pero teniendo en mente todos los aspectos positivos y negativos que esto implica. De esta manera, se pueden maximizar las fortalezas y oportunidades, y a la vez minimizar las debilidades y amenazas que se puedan enfrentar en el camino.

\section{El punto de partida y las preguntas iniciales}

La educación permanente y continua no sólo favorece y complementa la formación de profesionales graduados de las universidades públicas y privadas del país, sino que también se interesa por satisfacer las demandas e intereses del público en general, lo cual promueve una cultura de aprendizaje permanente, a través del desarrollo de actividades formales que responden oportunamente a las personas interesadas.

Por un lado, es responsabilidad de las universidades de este país el incrementar su oferta educativa, tanto de programas curriculares como de cursos, talleres y otras actividades abiertas al público. De manera que se apoyen procesos educativos donde se promueva la libertad y el desarrollo de capacidades y destrezas bajo el precepto fundamental: la educación es un bien público. Es decir, "la Educación y la Formación Continua" son los principales vórtices de identificación, pertenencia y promoción social a los que está llamada la academia. La educación permanente y continua es una plataforma que sostiene la formación a lo largo de toda la vida, es una herramienta de equidad, pues permite apropiarse de nuevos conocimientos y competencias.

Por otro lado, la vinculación externa remunerada se ha convertido, para la universidad, en una posibilidad para financiar sus actividades, especialmente en esta época debido a la disminución gradual del presupuesto público otorgado por el gobierno. Si bien, la Universidad de Costa Rica, desde sus inicios en 1940 hasta finales de 1970, registra actividades remuneradas, estas eran ocasionales. Según Meoño (2003), después de la crisis económica de 1979: 
O人ıмв Lu Wimblu, Rev. Estud. de Psicología UCR, 15(1) 2020 (Enero-Junio): 73-96 /ISSN: 1659-2107

La venta de servicios empieza a adquirir mayor valoración no sólo como fuente de ingresos en medio de la situación financiera universitaria, sino como mecanismo de transferencia de la UCR a la sociedad costarricense -en primer término- de tecnológica, y poco a poco de todo el conocimiento acumulado por esta casa de estudios superiores. (p.122)

En relación con lo anterior, históricamente, la venta de servicios mediante la educación permanente y continua ha significado la posibilidad de atenuar los efectos de tales decisiones y continuar produciendo lo que el país requiere, y que es resultado de la actividad académica. Por lo tanto, la vinculación externa remunerada no significa un fin en sí misma, sino más bien, la oportunidad de la universidad de acercarse, además de otras maneras, al contexto más inmediato, sea este nacional, regional o internacional. Esta vinculación con la comunidad facilita un intercambio de experiencias, expectativas, demandas e identificación. Estas a su vez, deben ser analizadas críticamente por la universidad y ser asumidas para dar respuesta según su competencia y responsabilidad.

Como muestra de la demanda que existe hacia las universidades públicas, durante el año 2013, la Universidad de Costa Rica desarrolló 288 proyectos de Educación Continua y Educación Permanente, muchos de los cuales fueron desarrollados con instituciones como el Ministerio de Educación Pública (MEP), el Ministerio de Ciencia y Tecnología (MICIT), el Instituto Costarricense de Electricidad (ICE), el Ministerio de Agricultura y Ganadería (MAG), Hospital de Niños, entre otras. En términos generales, se puede mencionar que los proyectos desarrollados en la Sección de Extensión Docente se vincularon con aproximadamente 200 organizaciones.

Durante el periodo 2014, en la Sección de Extensión Docente, se registraron un total de 319 proyectos bajo la modalidad de Educación Permanente. De ellos, 131 corresponden a Educación Continua (actualización profesional) y se destacan cuatro grandes áreas: Ciencias Sociales, Ingenierías, Artes y Letras y el área de Salud. En las Ciencias Sociales sobresale el grupo de Centros e Institutos (CCP, CICAP, CENTRO INFORMÁTICA, INIE, IIP, CIEP), en su conjunto representan más de 2000 certificaciones. Seguido, se encuentra la Facultad de Ciencias Sociales (Comunicación, Historia, Psicología, Trabajo Social) que llegó casi a los 1000 certificados. De igual forma sucede en el área de Ingeniería y Salud. En el primer caso, los Centros e Institutos INII, LANAMME y Centro de Informática representan 
Oेмив Lu Wimblu, Rev. Estud. de Psicología UCR, 15(1) 2020 (Enero-Junio): 73-96 /ISSN: 1659-2107

más de 2000 certificaciones entre aprovechamiento, participación, reconocimiento y asistencia; mientras que el segundo caso, los Centros e Institutos LEBI, CIHATA, y INISA suministran más de 600. En el área de Artes y Letras se destaca la Escuela de Filología, Lingüística y Literatura, la cual emitió, en ese año, más de 1500 certificados.

Ahora bien, todas las actividades registradas en ese periodo se encontraban relacionadas, principalmente, con temáticas como el fortalecimiento educativo dentro del cual se destaca la capacitación y la actualización profesional en todos los ámbitos, con mayor énfasis en algunas disciplinas. Asimismo, se rescata el enfoque de derechos humanos con proyectos dirigidos desde la atención de la niñez y la adolescencia hasta la población adulta mayor, el desarrollo ambiental sostenible en temas de gestión, seguridad e integración.

En el caso de la Sede de Occidente, las demandas de los pobladores de su área de influencia no son diferentes, por el contrario, desde hace muchos años se establece en los Planes Estratégicos la necesidad de crear un programa de educación continua y permanente como se establece en el Plan Estratégico Institucional 2015-2019. Este indica que una de sus metas es abrir un programa de educación continua en la sede para egresados y comunidad en general en áreas de actualización requeridas. Además, la Coordinación de Acción Social, ha recibido inquietudes y peticiones por parte de miembros de la comunidad con respecto a la oferta de cursos, talleres y diferentes actividades de capacitación, relacionadas con las áreas de salud, administración, informática e idiomas, ambiente, por decir algunas.

En esta línea, algunos procesos de acreditación, como el de la carrera de Bachillerato y Licenciatura en la Enseñanza del Inglés, identificaron la inconformidad de sus egresados por carecer de un programa de este tipo, el cual les dé la posibilidad de actualizarse constantemente. Igualmente, entre los resultados preliminares de los procesos de autoevaluación de carreras como Bachillerato y Licenciatura en Dirección de Empresas también están dando a conocer la necesidad de los egresados por actualizarse.

Lo anterior justifica la importancia de contar con un programa de este tipo en la Sede de Occidente, no sin antes considerar las políticas institucionales que justifican su apertura. De manera que las personas que participen del programa, a través de las actividades que se 
Oेмив Lu Wimblu, Rev. Estud. de Psicología UCR, 15(1) 2020 (Enero-Junio): 73-96 /ISSN: 1659-2107

realicen, podrán actualizar y adquirir nuevos conocimientos, así como desarrollar destrezas y competencias que les permita mejorar profesional o personalmente.

A su vez, la Universidad de Costa Rica y las comunidades establecen una relación de doble vía. La primera aporta sus conocimientos, su experiencia y se realimenta de un diálogo de saberes en beneficio de la docencia y la investigación, mientras que la comunidad recibe las capacitaciones necesarias acorde con sus necesidades. La población beneficiada entonces adquiere nuevos conocimientos, desarrolla destrezas y competencias para la vida y obtienen actualización profesional

Es así como finalmente el 10 de mayo del año 2016, la Comisión de Acción Social aprobó, de manera unánime, la formulación del Programa de Educación Continua de la Sede de Occidente, según acta número 284-2016 para que empezara a funcionar en enero del año 2017. La formulación fue presentada por la Coordinadora del Acción Social de ese entonces, la Dra. Milagro Piñeiro Ruiz, pero se le asignó a la docente del Departamento de Educación, Carolina Álvarez Fuentes, como responsable, y dos meses después se nombró a María Antonieta Vargas Solís como colaboradora, quien también estaba laborando para el Departamento de Educación.

Es así como el PECSO se crea con la intención de promover la educación permanente y continua en áreas académicas y técnicas ofrecidas en la Universidad de Costa Rica, principalmente en la Sede de Occidente, que permitan fomentar la adquisición y fortalecimiento de conocimientos, habilidades y destrezas de las personas de zona de influencia, en correspondencia con los retos y desafíos de la sociedad actual.

Dado lo anterior, esta sistematización tiene como objetivo dar a conocer los retos y logros del primer año del PECSO para lo cual se utilizaron las vivencias de la encargada y la colaboradora del programa, la formulación del proyecto ED-3280 Educacion Continua y el informe de labores. 
O人лвв Lu Wimblu, Rev. Estud. de Psicología UCR, 15(1) 2020 (Enero-Junio): 73-96 /ISSN: 1659-2107

\section{La recuperación del proceso vivido}

A partir del III Congreso Universitario celebrado en 1973, la acción social, en la Universidad de Costa Rica, representa el mecanismo mediante el cual se discute el quehacer institucional.

Consecuentemente, en 1974, se crea la Vicerrectoría de Acción Social (VAS) la cual se entiende como una extensión universitaria a partir del intercambio entre el centro universitario y la sociedad. Se posiciona la acción social como una actividad sustantiva, que, en conjunto con la investigación y la docencia, "contribuya a crear un ambiente favorable al cambio social y al fortalecimiento y creación de una auténtica cultura nacional. Corresponde también a la Universidad luchar por la superación de la dependencia cultural, contribuyendo al rescate de nuestros valores populares" (Universidad de Costa Rica, 1973). En este sentido, la universidad se asume en función de la sociedad costarricense, de las problemáticas y desafíos planteados y como agente dinamizador de cambio para el logro de las aspiraciones que busca la sociedad.

Según Angulo (s.f.), en la UCR la acción social se entiende como:

Una actividad sustantiva que vincula permanentemente a la Universidad con las comunidades, para establecer relaciones de mutuo aprendizaje que permitan en conjunto, lograr las transformaciones sociales necesarias para el mejoramiento de la calidad de vida y la realidad social en nuestro país. Esta alianza entra la academia y la sociedad, se realiza a partir de un marco ético, respetuoso de todos los saberes y que promueve la inclusión, la justicia y la solidaridad.

En sí, la universidad busca establecer una relación de índole social con la comunidad en la que el centro universitario se encuentra, donde se da un proceso de aprendizaje entre ambos sectores, pues aprende tanto la comunidad como la universidad. Por tanto, se considera que "la universidad debe incorporar al campo de su conocimiento y de su quehacer los problemas de la comunidad costarricense, para colaborar con aquellos sectores de ésta que más lo necesiten, es decir, fundamentalmente con los sectores populares" (Universidad de Costa Rica, 1981).

En los últimos años, la Sección de Extensión Docente de la Vicerrectoría de Acción Social de la UCR, se ha enfocado en la reflexión conceptual acerca de lo que significa la 
O人лмв Lu Wimblu, Rev. Estud. de Psicología UCR, 15(1) 2020 (Enero-Junio): 73-96 /ISSN: 1659-2107

Educación Permanente, denominada de esta manera por ser una definición más integradora. La Conferencia Mundial de Educación Superior realizada en París, en 1998, la considera como la actualización profesional y la capacitación para el desarrollo integral de las personas. Por lo tanto, de acuerdo con Agüero y Calderón (2013), la Educación Permanente integra:

La oferta educativa de actualización y capacitación dirigida a profesionales y a personas sin una formación académica pero que requieren del desarrollo y perfeccionamiento en sus competencias para el desarrollo del trabajo, así como considera aquellas acciones universitarias dirigidas al mejoramiento de la calidad de vida, a través de esas opciones de capacitación denominadas como educación para toda la vida.

Considerando lo anterior, en la educación permanente, como parte de la educación no formal, se considerarán todas aquellas actividades desarrolladas con y para la población con un fin educativo, sea esta de formación para el trabajo (a nivel profesional o técnico) o para el desarrollo de habilidades para la vida cotidiana. En este orden de ideas, la UNESCO (2011) establece que: "La educación no formal abarca las actividades educativas destinadas a la alfabetización de los adultos, la educación básica de los niños y jóvenes sin escolarizar, la adquisición de competencias necesarias para la vida diaria y competencias profesionales, y la cultura general".

Por lo tanto, se rescata la posibilidad del ser humano de aprender a lo largo de la vida, así como de la educación superior de ser una instancia facilitadora del proceso y no una mera transmisora de conocimiento. Es así como la Vicerrectoría de Acción Social de la Universidad de Costa Rica (s.f.) entiende la educación permanente y continua como:

Aquellas acciones educativas dirigidas al desarrollo del ser, para el mejoramiento de la calidad de vida, independientemente de la condición laboral. Son unas de las formas por las cuales la Universidad de Costa Rica pone a disposición de la población nacional su capacidad académica e institucional para brindar a personas de todas las edades y todos los niveles educativos, la oportunidad de seguir formándose en diversos conocimientos, habilidades y destrezas, para su desarrollo humano y profesional.

De este modo, la Sección de Extensión Docente entiende la educación para toda la vida como aquellas actividades de índole educativo encaminadas al desarrollo del ser, para 
el mejoramiento de la calidad de vida, independientemente de una condición laboral. Y educación continua, como aquellas actividades educativas que tienen por objetivo la actualización y la formación en competencias para el trabajo. Por lo que la Educación Continua (EC) se enfoca en actualizar tanto a los profesionales y a las personas sin formación académica, pero que requieren de capacitación para el desarrollo o perfeccionamiento en el desarrollo de un oficio o una actividad laboral. En ambos casos, la oferta de EC se brinda en el marco de la vinculación con la organización a la cual pertenece la persona trabajadora, o bien como una oferta más amplia en la cual se participa de manera individual.

De acuerdo con las políticas institucionales, uno de los temas de interés es la EC, particularmente, la actualización profesional, enfocada tanto a lo interno como hacia lo externo de la universidad. Asimismo, en las políticas del periodo comprendido entre el 20102014 y vigentes al 2015, así como las del 2016, se plantea el fortalecimiento de la capacitación del personal docente y administrativo, la promoción de iniciativas para el dominio de idiomas y tecnologías necesarias en el desarrollo laboral, así como la disposición del conocimiento académico al servicio de la sociedad, donde la actualización vendría a ser un complemento necesario para el evolutivo ámbito laboral. En este intercambio de saberes, de la universidad con la sociedad, la primera colabora con el desarrollo de la sociedad y ésta a la vez realimenta su quehacer.

Para el caso de la Sede de Occidente de la Universidad de Costa Rica, en 1975, se crea la Coordinación de Acción Social (Piñeiro, 2018). En ese momento, se planificaron, organizaron y desarrollaron diversas actividades, entre ellas: cursos, talleres, conferencias y simposios, como respuesta a intereses y necesidades planteados por diferentes organizaciones e instituciones, tales como el Ministerio de Educación Pública y la Caja Costarricense de Seguro Social, pero no se inició ningún programa dedicado a la EC.

De esta manera, desde hace más de cuarenta años, se han desarrollado acciones enfocadas en desarrollar procesos de enseñanza y aprendizaje para la formación y capacitación continua de personas, desde los diferentes departamentos y coordinaciones de la Sede de Occidente. Es en el año 2014, cuando la Dra. Piñeiro asume la Coordinación de Acción Social y ve reflejada, en los objetivos y metas del Plan Estratégico Institucional 20152019 de la Sede de Occidente, la necesidad de formular un Programa de Educación Permanente y Continua. 
A partir de lo expuesto, en mayo de 2015, se hace un primer intento de impartir cursos de idiomas que respondieran a las necesidades de educación continua y permanente. Se elaboraron cuatro programas de idiomas extranjeros entre inglés y portugués. De estos, se impartieron tres. Los cursos de idiomas se establecieron con una duración de 40 horas cada uno y se matricularon de entre 18 y 20 personas en cada uno. Para el mes de setiembre de 2016, se elaboró el programa de Inglés Conversacional con una duración de 40 horas, con un horario de 4 horas semanales, y se ofreció a personas cuyo nivel de inglés fuera intermedio o avanzado.

Aunado a ello, y como resultado de los procesos de introspección de la Sede de Occidente (SO), se refleja la necesidad de disponer de un proyecto que sistematice y ofrezca, a la vez, nuevos conocimientos y destrezas a las poblaciones profesionales y no profesionales del área de influencia de la sede. Debido a lo anterior, la SO inscribe el Programa de Educación Continua de la Sede de Occidente (PECSO) ante la Vicerrectoría de Acción Social (VAS) e inicia labores en enero del año 2017.

Desde ese momento, el proceso de trabajo del PECSO se visualizó como un ciclo, de manera que las acciones y decisiones se relacionaran entre ellas y se realizara una evaluación constante de los resultados para que el programa se fuera adaptando su oferta a la cambiante demanda de la sociedad que beneficia. De esta manera se establecieron 5 etapas de planificación iniciando con el diagnóstico y concluyendo el ciclo con la elaboración del informe final.

En la primera etapa, se revisó una aproximación de diagnóstico de las necesidades de educación permanente y continua y las áreas temáticas, requeridas por las personas del área de influencia, para poder corresponder a sus necesidades reales, así como a las necesidades de los egresados de la sede. Se utilizaron diferentes fuentes de información tales como:

- La investigación el "Fortalecimiento del vínculo de la Sede de Occidente con la Comunidad", realizada por Barrantes, Palma, Romero y Solano. Dicho estudio identificó las necesidades empresariales existentes en la zona de Occidente.

- Los resultados del proceso de Autoevaluación de la Carrera Bach. y Lic. en la Enseñanza del Inglés. Este documento muestra las necesidades en formación y 
actualización que tienen los egresados de la UCR, SO, y también las demandas de formación establecidas por los empleadores de las personas graduadas de esta carrera.

En la segunda etapa, se esperaba llevar a cabo reuniones con los directores de los Departamentos de carrera de la Sede de Occidente y con coordinadores de carreras para intercambiar ideas de posibles cursos que se podrían ofertar y de esta manera subsanar las necesidades de los egresados de la sede.

La tercera etapa se trata de planificación. De acuerdo con las solicitudes recibidas o necesidades detectadas, se programaron cursos, se estableció la cantidad de horas para cada una de las actividades, así como el costo por concepto de matrícula y el salario del profesor. Como parte de este proceso, también se llevaron a cabo las inscripciones de cada actividad ante la Vicerrectoría de Acción Social, la revisión de los programas de cada uno de los cursos, la divulgación de las actividades por medios digitales, el proceso de matrícula de la actividad, la adquisición de materiales, darles seguimiento a las actividades, evaluarlas al finalizar estas y solicitar, tramitar y entregar los certificados de aprovechamiento, participación o asistencia según correspondía y bajo los lineamientos de la Dirección General de Servicio Civil (2013).

En la cuarta etapa, se llevó a cabo la evaluación y un breve análisis de las actividades realizadas. Para ellos, al finalizar cada actividad, se solicitó a los participantes llenar un cuestionario de forma digital cuyo enlace fue enviado a los correos electrónicos. Con esta evaluación se buscaba obtener información acerca del grado de satisfacción del curso, del profesor y del apoyo administrativo del PECSO.

La quinta etapa se entrelaza con la anterior, pues tiene que ver con la elaboración del informe anual de labores, la cual representa la evaluación del programa como tal. En primera instancia, el PECSO fue evaluado por la encargada de la Sección de Extensión Docente, luego por la Comisión de Acción Social y finalmente por la Vicerrectoría de Acción Social.

Tanto la encargada del programa como la colaboradora, de ese entonces, elaboraron y revisaron el informe final en el que se incluyó información general, balance (cumplimiento de objetivos, las etapas cíclicas de trabajo, los logros, la vinculación con otros proyectos de Acción Social y limitaciones), seguimiento, evaluación y divulgación, así como la información financiera. Es con base en dicho documento que se lograron determinar los retos y logros que tuvo el programa en su primer año. 
O人ıмв Lu Wimblu, Rev. Estud. de Psicología UCR, 15(1) 2020 (Enero-Junio): 73-96 /ISSN: 1659-2107

\section{Reflexiones de Fondo}

Para interpretar la dinámica del PECSO durante su primer año de trabajo, hay que examinar el proceder de sus componentes, preguntarse por las causas de lo sucedido para hallar los factores principales que describan las razones por las cuales las situaciones se dieron de cierto modo (Jara, 2011). Así las cosas, se tomaron en cuenta los siguientes aspectos: ubicación geográfica, población meta del proyecto, los objetivos del programa y asuntos de gestión administrativa. Cabe aclarar que no todos estos aspectos se pueden evaluar de manera cuantitativa.

\section{Ubicación Geográfica}

En la formulación del proyecto se tenía proyectado impartir cursos en el Museo Regional de San Ramón el cual se ubica en el distrito central de San Ramón, pero luego se tuvo que cambiar de sede. En un principio se ofrecieron cursos de inglés para beneficiar a la población que depende del transporte público, sobre todo de los autobuses. Sin embargo, a pesar de que se ofertaron los cursos, no se logró matricular un mínimo de estudiantes. Lo cual significa que la cercanía con el transporte público no representa un factor decisivo para las personas interesadas en matricular cursos con el PECSO; por lo tanto, este no debería ser un punto que considerar en la planificacion de cursos.

Como resultado de lo sucedido, a partir de agosto los cursos se siguieron impartiendo solamente en la Sede de Occidente ubicada en el distrito de Alfaro. Se hizo uso de aulas regulares y del Módulo de Trabajo de Acción Social. Asimismo, a petición de COOPEALFARORUIZ, el PECSO llegó a Zarcero para impartir cursos de inglés básico para sus empleados, ampliando de esta manera el área de cobertura del programa.

\section{Población Meta del Proyecto}

En la formulación del proyecto, se estimó beneficiar alrededor de 500 personas de forma directa en la región de Occidente, tales como profesionales, egresados, y estudiantes avanzados de carreras universitarias. Así también, organizaciones, grupos organizados o instituciones públicas y privadas que requirieran de cursos, talleres u otras actividades 
O人ıмв Lu Wimblu, Rev. Estud. de Psicología UCR, 15(1) 2020 (Enero-Junio): 73-96 /ISSN: 1659-2107

relacionadas con la educación permanente y continua. Para beneficio de la zona de influencia de la universidad, en vez de 500 se atendieron 600 personas, lo cual significa un incremento de un $20 \%$ sobre la cantidad inicialmente estimada.

A su vez, tanto la colaboradora como la encargada del PECSO pudieron valorar cuantitativamente los cambios en la población que se atendió. Esto por cuanto, en los cursos de aprovechamiento (en los cuales hay evaluación sumativa) el $99 \%$ de los estudiantes aprobaron el curso. Eso demuestra que la población beneficiada culminó cada curso con un mayor bagaje de conocimientos y destrezas. La mayoría de ellos asumen el reto de llevar un curso con mucha responsabilidad y conscientes de que van a aprender o perfeccionar una destreza, incrementar su saber en el ámbito profesional y ampliar su currículum. Todo lo anterior crea profesionales más preparados y con mayores posibilidades de escalar en el ámbito laboral.

Asimismo, se logró alcanzar a población considerada no profesional con los cursos de costura, lo cual calza perfectamente con la definición de educación permanente. Estos beneficiaron mayoritariamente a mujeres con un perfil distinto al estudiante o egresado universitario quienes a su vez mostraron satisfacción por llevar cursos a un costo muy módico y cuyos conocimientos adquiridos eventualmente podrían convertirse en una fuente de trabajo, pues aparte de la costura también aprendieron sobre cómo realizar cálculos de costo de materiales y mano de obra.

\section{Objetivos del Programa}

El PECSO se formuló y se encaminó a trabajar en 3 objetivos específicos para su primer año de trabajo.

Objetivo 1: Diagnosticar las áreas temáticas y necesidades de educación permanente y continua requeridas por instituciones, organizaciones, grupos organizados o personas de la comunidad, que permitan desarrollar cursos, talleres, conversatorios, simposios, desde las secciones de carrera y responder a necesidades reales.

En relación con este objetivo, cuando se formuló el PECSO se hizo un diagnóstico inicial basado en el compromiso de mejora de la carrera de Bach. y Lic. en la Enseñanza del Inglés, de la investigación "Diagnóstico de expectativas que tienen los estudiantes del último año de Secundaria de la Dirección Regional de Educación de Occidente acerca de la Educación 
O人лмв Lu Wimblu, Rev. Estud. de Psicología UCR, 15(1) 2020 (Enero-Junio): 73-96 /ISSN: 1659-2107

Superior" (Alfaro, Piñeiro y Quesada, 2016) y de la ponencia "Seguimiento a la población egresada de la carrera Bachillerato y Licenciatura en la Enseñanza del Inglés (2012-2015)" (Araya, Chan, y Córdoba, 2016). Como resultado de este diagnóstico en la segunda quincena de enero del 2017 se inició con la oferta de cursos de inglés.

A la vez, debido a que tanto la encargada del PECSO como la colaboradora son docentes de inglés se decidió aprovechar su formación académica para mejorar los cursos de este idioma que anteriormente estaban a cargo de la oficina de Extensión Docente. Es así como dichos cursos se organizaron en 12 módulos, siendo cada curso de aprovechamiento y de 40 horas de acuerdo con los lineamientos del Servicio Civil. Sin embargo, el cambio más significativo fue utilizar el libro Four Corners de Richards and Bohlke. Esto por cuanto dicho libro está actualizado y basado en el Marco Común Europeo de Referencia (CEFR) de idiomas, logrando llevar al estudiante a alcanzar el nivel B1.

Por otra parte, la encargada del programa se reunió con el director del Conservatorio de Música de la Sede de Occidente (CMO) con el propósito de determinar las necesidades que esa población. Como resultado de ese encuentro se inscribió el Campamento de Piano y se propuso un curso de canto para el año siguiente.

En este orden de ideas, a inicios del I ciclo 2017, la encargada del PECSO se reunió con la encargada del Proyecto de Actualización y Educación Continua para Docentes de Educación Primaria de los Centros Educativos de la Región de Occidente. Este proyecto pertenece a la Sección de Educación Primaria del Departamento de Educación de la SO. En esa reunión, se socializaron los resultados del diagnóstico de necesidades de los docentes de primaria. Entre las necesidades más importantes se hacía necesario actualizar a los docentes en cuanto al Reglamento de Evaluación de los Aprendizajes y la aplicación del Debido Proceso. Gracias a este enlace entre programas, de la misma sede, se logró que el PECSO ofertara los cursos.

También, la encargada del programa se reunió con miembros de la Asociación de Estudiantes de la carrera Bach. y Lic. en la Enseñanza del Inglés. En esa reunión, los estudiantes propusieron crear un club de inglés conversacional para estudiantes de inglés que estaban iniciando la carrera, para funcionarios de la universidad y personas de la comunidad. Los clubs se inscribieron como cursos de aprovechamiento y se reunían 2 horas semanales durante 16 semanas. Esto representó un logro para el PECSO, pues de manera totalmente 
O人ıмв Lu Wimblu, Rev. Estud. de Psicología UCR, 15(1) 2020 (Enero-Junio): 73-96 /ISSN: 1659-2107

gratuita se daba la oportunidad a las personas de tener un título bajo los lineamientos del Servicio Civil.

Además, en el segundo semestre del 2017, la encargada del programa se reunió con el coordinador y dos docentes de la carrera de Bach. y Lic. en Dirección de Empresas. En la reunión, los docentes expusieron la necesidad de ofrecer un curso de inglés específico para la carrera. A causa de esto, se publicó un afiche en la página de Facebook del PECSO para invitar a los interesados en llevar los cursos a participar en un diagnóstico lingüístico y establecer su nivel de inglés.

Con base en los resultados del diagnóstico, se diseñaron los cursos de Business English con 6 cursos modulares. Los cursos se inscribieron bajo la categoría de aprovechamiento con una duración de 40 horas. Cabe aclarar que, aunque en primera instancia, los cursos estaban pensados en los egresados de la carrera, no fue posible contratar a un docente de inglés que tuviera tanto disponibilidad de horario como conocimientos en inglés con fines específicos, por lo que los cursos se ofrecieron a los estudiantes de tercer año de la carrera. De esta manera se logró que los participantes aprendieran dicho idioma en el contexto laboral haciendo su aprendizaje más significativo.

Desgraciadamente, los cursos debieron cerrarse debido a que los estudiantes debían pagar el costo y libro del curso y muchos de ellos eran becados. También, los estudiantes manifestaron que debido a que el curso de inglés no era parte de la malla curricular de la carrera, la carga académica se les incrementaba.

Por otra parte, la coordinadora de Acción Social de la SO y la encargada del PECSO, se reunieron con diferentes profesionales para valorar el interés en apoyar al PECSO desde sus áreas de experiencia, por medio de la oferta de cursos. Se propusieron 3 cursos de danza contemporánea (uno para adultos, otro para jóvenes y el último para adolescentes), 2 cursos de fotografía experimental, 2 cursos de diseño en 3D, 1 curso de redacción básica y cursos de creación y expresión corporal para personas adultas mayores.

Todos los cursos se ofertaron, pero no todos se impartieron debido a que no se matricularon el mínimo de estudiantes necesarios para costear el costo del docente encargado de cada curso. Esto nos lleva a pensar que, aunque un curso parezca ser una buena idea, es la población quien al final determina si el curso es de su interés o no. 
Por otro lado, la señora Álvarez, encargada del PECSO, se reunió con una técnica especializada en diseño de modas para inscribir cursos de costura para subsanar las necesidades de aprendizaje de un grupo de mujeres en la zona de San Ramón. Se ofreció Costura Básica, Técnicas de Ensamble y Patronaje para Ropa de Patronaje de Camisas y para Dama. Estos cursos al igual que los cursos de inglés eran de aprovechamiento, pero con una duración de entre 50 y 64 horas.

Lamentablemente y a pesar de la acogida, estos eventualmente se dejaron de ofertar debido a que, al no ser propuestos desde una sección de carrera, la universidad no cuenta con máquinas de coser ni las condiciones idóneas para impartir este tipo de cursos.

Por último, la encargada y colaboradora del PECSO organizaron una reunión con la encargada del Centro de Investigaciones de la Mujer (CIEM), una trabajadora social del Ministerio de Salud Pública y la encargada de Extensión Docente de la SO para planear el curso "Sentir, pensar y enfrentar violencia intrafamiliar". Este curso se ofrecería en marzo del 2018, de manera gratuita, con el fin de capacitar a organismos gubernamentales y no gubernamentales que hacen el primer contacto con poblaciones que denuncian ser víctimas de violencia. Se inscribió bajo la categoría de aprovechamiento y se impartió de manera exitosa en el Museo Regional de San Ramón.

Dado todo lo anterior, aunque el diagnóstico de necesidades no se llevó a cabo desde las secciones de carrera, debido a la limitación de tiempo de la encargada y la colaboradora del PECSO para concretar reuniones con los coordinadores de cada carrera, el objetivo 1 se cumplió en un $100 \%$, pues para medirlo se tenían como metas contar con un diagnóstico y llevar a cabo al menos 2 reuniones con jerarcas de instituciones, organizaciones y empresas públicas y privadas.

El diagnóstico no-formal para detectar necesidades de educación permanente y continua se realizó tanto con docentes de diferentes carreras de la SO, como con profesionales fuera de la universidad, cumpliendo de esta manera con la meta. A la vez, la meta relacionada con la cantidad de reuniones se sobrepasó en un $250 \%$ al llevarse a cabo 7 reuniones en vez de 2. Por lo tanto, se puede afirmar que en el año 2017 se atendieron las necesidades de educación continua y permanente que fueron expuestas ante el PECSO.

Objetivo 2: Promover desde las secciones de Carrera la creación de cursos, seminarios, talleres, etc. que favorezcan los procesos de educación permanente y continua dirigidas a las 
personas de las comunidades y a la comunidad universitaria, que permitan el fortalecimiento académico y desarrollo profesional y social.

Acerca de este objetivo, en el segundo mes de funciones del PECSO, la encargada se reunió con el director del Departamento de Ciencias Naturales de la SO para conversar sobre las posibilidades de que los profesores de las diferentes carreras del departamento sugirieran cursos para impartir a sus egresados por medio del PECSO. Empero, ningún docente envió propuestas. También, se conversó sobre la posibilidad de crear un Programa de Formación en Recurso Hídrico, pero faltó seguimiento y nunca se concentró.

Igualmente, los profesores de la sección de la carrera de Bach. y Lic. en la Enseñanza del Inglés fueron invitados vía oficio a incorporarse a colaborar con los cursos de inglés del PECSO. No obstante, ninguno respondió. Consecuentemente, la falta de propuestas presentadas por los mismos docentes de la sede representa un reto para el programa, pues son ellos quienes mejor conocen las necesidades de educación continua de sus egresados.

Por otro lado, en el campo de la educación e idiomas se ofrecieron cursos de coreano, japonés y portugués básico. Por su parte, con el apoyo de docentes de las secciones de educación primaria y secundaria de la SO, se impartieron tres talleres de participación sobre la nueva metodología de inglés (Action-Oriented Approach) implementada por el Ministerio de Educación Pública (MEP). Los talleres fueron dirigidos a docentes del Depto. de Educacion de la SO, a docentes en ejercicio del MEP y a recién egresados de la carrera de Bach. y Lic. en la Enseñanza del Inglés.

En definitivo, el objetivo 2 se cumplió en un 100\%, pues para medirlo se tenía como meta contar con al menos 6 programas de cursos o talleres. Aunque varios de los cursos no se promovieron desde las secciones de carrera, sí correspondían a temas de interés para personas de las comunidades.

En este sentido, es claro que beneficio que llevó el PECSO a la comunidad, pues en su primer año se ofertaron 21 cursos en diferentes áreas tales idiomas, educación, artes gráficas, danza y moda.

En la Figura 1 se muestran algunos de los afiches que se postearon en la página de Facebook del PECSO para promocionar los cursos anteriormente mencionados. 

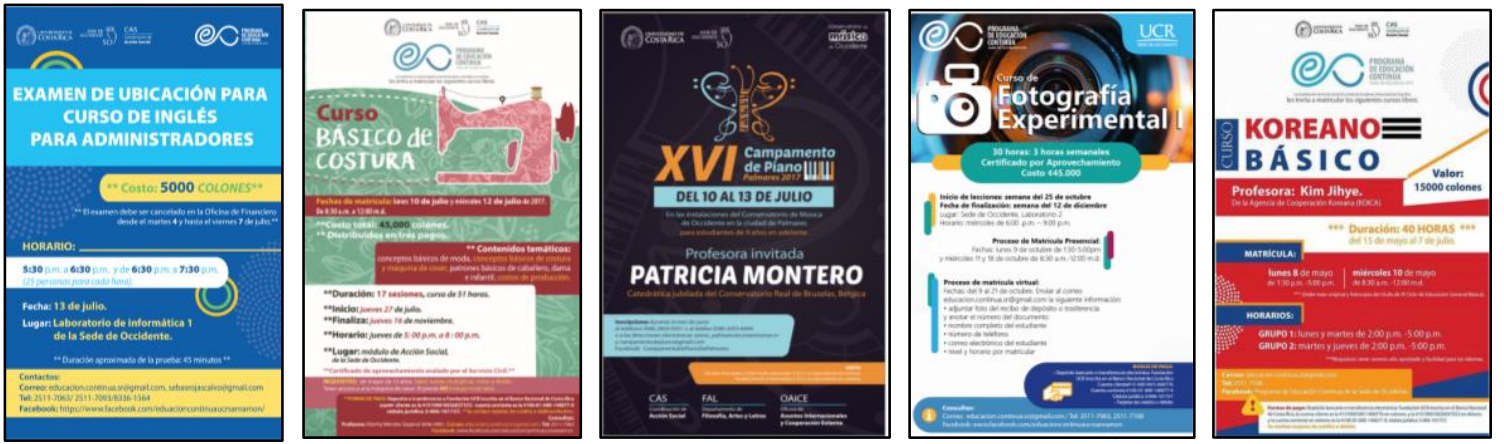

Figura 1. Ejemplos de afiches de cursos ofertados por el PECSO Fuente: Programa de Educación Continua de la Sede de Occidente, 2017

Objetivo 3: Implementar desde el programa los cursos y actividades tales como: congresos, simposios, foros, mesas de discusión, entre otros, que permitan promover la educación permanente y continua en las personas interesadas.

En relación con este objetivo, el PECSO inscribió, colaboró con la coordinación de IV Seminario Carlos Monge Alfaro y gestionó la elaboración de los certificados para los ponentes del seminario. El seminario tuvo una participación de 34 ponentes en total.

De la misma manera, el PECSO inscribió el I Encuentro Interuniversitario de Saxofonistas de la Sede de Occidente ante la Vicerrectoría de Acción Social, apoyó con la logística organizativa y gestionó la elaboración de los certificados para los saxofonistas internacionales.

Conjuntamente desde el programa mismo, el PECSO implementó más cursos de los propuestos en la formulación de este. Por lo tanto, el nivel de logro del objetivo 3 depende desde la perspectiva con la que se analice. Para medirlo se tenía como meta desarrollar al menos 6 cursos, 2 talleres y un conversatorio.

Si se toma en cuenta que no se llevó a cabo ningún conversatorio, el objetivo se logró en un $88 \%$. No obstante, si se analiza que se ofertaron 21 cursos en vez de 6 , esto significa que la meta se sobrepasó en un $249.9 \%$. A su vez, si se piensa en los talleres, la meta se sobrepasó en un $50 \%$ pues se ofrecieron 3 talleres en vez de 2 . 
O人лмв Lu Wimblu, Rev. Estud. de Psicología UCR, 15(1) 2020 (Enero-Junio): 73-96 /ISSN: 1659-2107

\section{Gestión Administrativa}

En cuanto a la gestión administrativa esta debe entenderse como aquellas actividades que la encargada y la colaboradora del PECSO llevaron a cabo para administrar el programa desde la apertura de este. En este sentido, uno de los retos más grande fue determinar la mejor forma de darse a conocer entre la población del área de influencia de la SO. Inicialmente, se trató de enterar a la comunidad de la existencia del programa mediante la colocación de afiches, pero después de alrededor de dos meses de iniciado el programa, se determinó que esto implicaba invertir mucho tiempo en la movilización e incurrir en otros gastos como el transporte para encontrar sitios apropiados para la publicidad.

Sin embargo, este reto dio pie a un gran logro, el cual fue crear y mantener activa la página de Facebook del PECSO. Gracias a esta herramienta y también a las publicaciones hechas en la página oficial de la Sede, la convocatoria de matrícula de los cursos tuvo un mayor alcance y la comunicación con personas interesadas fue más fluida. Es así como se le anunció a la comunidad de Occidente la fecha de matrícula de inscripción de los cursos, modos de pago y fechas importantes.

De la misma manera, los procesos de gestión administrativa que implica iniciar un programa de este tipo demandan más tiempo del que se le asignó a la encargada y a la colaboradora (10 horas semanales a cada una), pues no contaban con secretaria ni asistente. Aunado a esto y a la importancia de tener estudios formales en administración, no se asignaron tareas específicas a cada una de ellas, disminuyendo la eficacia en el uso de las horas de trabajo, ya que entre ambas se atendían inscripciones de los cursos ante la Vicerrectoría de Acción Social, solicitud de certificados, actualización de la página de Facebook, lectura y respuesta de correos electrónicos, procesos de matrícula, etc.

Para este último punto había que inscribir manualmente a cada estudiante en el curso escogido e ingresar los datos de la persona en una hoja de Excel, luego ingresar los datos en el sistema de facturación de la fundación para crear una factura, imprimirla o enviarla por correo electrónico.

En este orden de ideas el cómo y dónde organizar la información del PECSO resultó ser un dilema importante. Es así como se decidió crear un Drive para guardar, compartir y acceder de forma más fácil la información relacionada con el programa. Empero, debido a 
la limitación de tiempo no siempre era posible organizar los datos en carpetas y ocasionalmente se dificultaba encontrar la información.

En particular, cuando se escribía un oficio se guardaba cada uno como un documento aparte. Esto significaba que cada vez que se ocupaba escribir otro había que buscar el último para continuar la numeración y no siempre era fácil de localizar. Luego se decidió hacer un solo documento con todos los oficios, lo cual facilitó la localización de la información.

Por otro lado, a pesar del apoyo recibido por parte de la asesora de proyectos de Extensión Docente y del ejecutivo de la Fundación UCR, al inicio no se tenía conocimiento que las actividades debían ser previamente inscritas ante la Vicerrectoría de Acción Social. Además, tanto la encargada como la colaboradora requerían conocer de normativas, lineamientos y reglamentos relacionados con el vínculo externo, servicio civil, extensión docente y de la fundación misma y todos estos procesos y la asimilación de la información debían concomitase. Esto representó un gran reto pues había que digerir una considerable cantidad de información en un corto tiempo.

Otro reto para la administración del programa fue no disponer de un espacio físico y recursos (computadora, impresora, teléfono) directamente asignados al PECSO. Esta limitante se sobrellevó mediante la solicitud que se le presentó a los compañeros de oficina y la Coordinación de Acción Social quienes eventualmente facilitaron áreas de sus espacios de trabajo y equipo de oficina para cederlos al programa. No obstante, solamente se entregó una computadora portátil y cuando la encargada y la colaboradora estaban trabajando al mismo tiempo evidentemente no había equipo para ambas.

Por otra parte, otro reto tiene que ver con la contratación de docentes ya que se torna un campo difícil de navegar. Los lineamientos para el vínculo remunerado estipulan que los docentes de la Universidad de Costa Rica son quienes deben impartir los cursos ofrecidos a la comunidad, o al menos tener prioridad. Sin embargo, como se mencionó anteriormente, los docentes que trabajan para la universidad no tienen disponibilidad horaria o gozan del beneficio de dedicación exclusiva, por lo que no pueden laborar para el proyecto.

Por lo que, una solución fue contratar egresados y así impartir cursos por personal altamente capacitado, pero que no labora para la universidad. A su vez, algunos cursos necesitaban de un docente con un perfil muy específico que no es fácil de conseguir. Por lo que, en algunas ocasiones, los cursos no se pudieron ofertar. 
Por último, estimar los costos para cada actividad tiene un cierto grado de dificultad, ya que los programas de educación continua "pueden tener un vínculo remunerado con la sociedad o bien ser una contribución gratuita a los diversos sectores con principal acento en aquellos que se ven vulnerabilizados” (Agüero, Calderón, Granados, Rímola, y Sánchez, 2014). Es así como otro logro del PECSO fue ofrecer cursos con costos muy accesibles para la población beneficiada pues, concretamente, los clubs de inglés se dieron $100 \%$ gratuitos. A su vez, se ofrecieron diferentes formas de pago dependiendo del curso y la población meta. Algunos cursos se cancelaban de manera mensual y otros en un solo pago. Es decir, el costo del curso variaba, así como su forma de pago ya que el PECSO no tiene fines de lucro.

\section{Puntos de llegada}

Con la creación del Programa de Educación Continua de la Sede de Occidente, la sede logra cumplir con una de las metas principales del Plan Estratégico 2015-2019 y a la vez convertirse en una plataforma al servicio de las carreras universitarias para responder a sus necesidades emergentes como resultado de sus procesos de autoevaluación en miras a la acreditación. Sin embargo, lo más importante es que por medio del programa tanto graduados universitarios como no universitarios tuvieron la oportunidad de actualizar su conocimiento desde la arista social de la universidad, cuyo propósito central es generar conocimientos para transformar, profesionalizar y capacitar a la población del área de influencia.

Asimismo, como resultado de esta sistematización es importante reflexionar sobre los retos y logros del primer año del PECSO para aprender de lo sucedido y compartir con otros que pueden estar inmersos en un proceso similar, pero para llevar a cabo este tipo de análisis hay que tener muy claro cuáles son los objetivos del programa, qué busca cada uno de ellos, trabajar de la mano con ellos y trazar una ruta clara para alcanzarlos sin perder el norte.

En definitivo el reto más grande del PECSO fue la parte de gestión administrativa, por lo cual es imperativo tener comunicación fluida con los asesores de Extensión Docente de la Vicerrectoría de Acción Social y de la Fundación de la UCR, pues su acompañamiento es vital para el buen funcionamiento de un programa de este tipo y las actividades de vínculo externo. De esta manera se evitarán posibles retrasaron involuntarios de gestiones administrativas. 
En un comienzo es una buena idea dirigir esfuerzos en familiarizarse con la parte administrativa y programar la cantidad de actividades que sean manejables para las personas encargadas y que además estas sean basadas en algún tipo de diagnóstico para incrementar el grado de apertura y éxito de las actividades. De esta manera como se dice popularmente "menos es más".

A su vez es importante entender que, aunque es un programa de acción social hay que visualizarlo como una pequeña empresa que debe conocer bien a su población meta. Por lo que hay que hacer un estudio de mercado o un diagnóstico formal para conocer las prioridades de las personas, instituciones y organizaciones del área de influencia.

Con base en ello, se pueden valorar si se toman en cuenta aspectos como el transporte público o el lugar donde se imparten los cursos como un parámetro para planificar actividades. Esto asegurará que lo que se oferta cumpla con las expectativas y necesidades de los interesados ya que lo que los encargados del programa consideren una prioridad o una temática importante no necesariamente es lo que la población necesita. Esto a su vez logrará una mayor correspondencia entre la oferta y lo que realmente se imparte.

Por otro lado, el logro más importante del PECSO fue la cantidad de cursos y actividades que se ofertaron, ya que aparte de la cantidad, se abarcaron diferentes temáticas y poblaciones. Los cursos para la población no universitaria fueron los que tuvieron más aceptación por lo que hay que continuar sirviéndole a este público con cursos de educación permanente.

De la misma manera, para promover cursos desde las secciones de las carreras universitarias hay que motivar principalmente a los docentes interinos que no tienen dedicación exclusiva o a los propietarios con mayor disponibilidad de horario, pues de lo contrario se tendrá que continuar trabajando con egresados. Para facilitar este punto se recomienda hacer un banco de datos de egresados que probablemente las mismas carreras puedan facilitar.

También, trabajar de la mano con los coordinadores de carrera de la sede y de otras sedes podría ayudar a planificar actividades formales como congresos, simposios, foros, etc. De esta manera toda la población de la universidad y no solamente la de una sede se vería beneficiada. 
O人ıмв Lu Wimblu, Rev. Estud. de Psicología UCR, 15(1) 2020 (Enero-Junio): 73-96 /ISSN: 1659-2107

En conclusión, al inicio de todo programa, los retos son probablemente mayores, pero es una cuestión de conocer las necesidades e intereses de la población de la zona de influencia, solicitar ayuda tanto a los docentes y autoridades de la universidad, tener en mente el fin social del programa, ser perseverantes y sobre todo utilizar principios fundamentales de administración para alivianar la carga de trabajo y gestionar de manera eficiente y eficaz.

\section{Referencias}

Agüero Hernández, Heiner y Calderón Valverde Rosalba. Los retos de la Educación Continua en la Universidad de Costa Rica. Conferencia presentada en la pasantía en la Universidad Autónoma de Chiapas, Chiapas, México, 2013.

Agüero Hernández, Heiner, Calderón Valverde Rosalba, Granados Castro, Paula, Rímola Obregón, Johanna, Sánchez Fallas, Marilín. Inscripción y funcionamiento de los Programas y Proyectos de Educación Continua y Servicios Especiales Ponencia. Ponencia presentada en el VII Congreso Universitario, Universidad de Costa Rica, Costa Rica, 2014.

Alfaro Valverde, Alicia, Piñeiro Ruiz, Milagro, Quesada Rojas, Óscar. Diagnóstico de expectativas que tienen los estudiantes del último año de secundaria de la Dirección Regional de Educación de Occidente, acerca de la educación superior., 2016.

Angulo Ugalde, Yamileth, s.f., Vicerrectoría de Acción Social. ¿Qué es Acción Social? Universidad de Costa Rica, s.f., acceso el 24 de marzo del 2020, https://accionsocial.ucr.ac.cr/quienes-somos

Araya Araya, Karla, Chan Díaz, José, Córdoba Gonzalez, Gustavo. Seguimiento a la población egresada de la carrera Bachillerato y Licenciatura en la Enseñanza del Inglés (2012-2015): principales resultados y retos. Ponencia presentada en el IV Coloquio Investigación Psicológica y Educativa, Universidad de Costa Rica Sede de Occidente, Costa Rica, 2016

Dirección General de Servicio Civil. RESOLUCION DG-135-2013. San José, Costa Roca: Dirección General de Servicio Civil, 2013.

Jara Holliday, Óscar. (2011). Orientaciones teórico-metodológicas para la sistematización de experiencias. Biblioteca Electrónica sobre Sistematización de Experiencias. Disponible en: http://cepalforja.org/sistematizacion 
O人лмв Lu Wimblu, Rev. Estud. de Psicología UCR, 15(1) 2020 (Enero-Junio): 73-96 /ISSN: 1659-2107

Meoño Molina, Rita. Vinculación Remunerada con el Sector Externo: Un reto para la Facultad de Ciencias Sociales de la Universidad de Costa Rica. Tesis para optar por el grado de Maestría en Trabajo Social. Universidad de Costa Rica, San José, Costa Rica, 2003.

Piñeiro Ruiz, Milagro. La acción social en la región de Occidente: logros y proyección. San Ramón, Alajuela, Costa Rica: Universidad de Costa Rica, Sede de Occidente, Coordinación de Acción Social, 2018.

UNESCO. Glosario del Informe de Seguimiento de la Educación para todos en el Mundo. http://www.unesco.org/new/fileadmin/MULTIMEDIA/HQ/ED/pdf/gmr2011glossary-es.pdf. 2011

Universidad de Costa Rica. Resoluciones definitivas III Congreso Universitario. San José, Costa Rica, Universidad de Costa Rica, 1973

Universidad de Costa Rica. Una proyección de la Universidad al servicio de las comunidades. San José, Costa Rica: Universidad de Costa Rica, 1981.

Vicerrectoría de Acción Social. Educación Permanente y Continua, s.f., acceso el 24 de marzo del 2020, https://accionsocial.ucr.ac.cr/educacion-permanente-y-continua 